\title{
Comparison of Various Compressible Vorticity Confinement Methods and Development Two New Confinement Parameters
}

\author{
H. Bagheri Esfe ${ }^{1 \dagger}$, M. Malek Jafarian ${ }^{2}$ and M. Bagheri Esfe ${ }^{3}$ \\ ${ }^{1,3}$ Department of Mechanical Engineering, Amirkabir University of Technology (Tehran Polytechnic), Tehran, \\ 15875-4413, Iran \\ ${ }^{2}$ Department of Mechanical Engineering, Birjand University, Birjand, Iran \\ †Corresponding Author Email: hamedbagheriesfe@gmail.com
}

(Received August 30, 2010; accepted March 27, 2011)

\begin{abstract}
In this paper, vorticity confinement parameters are successfully developed for compressible flows. The first new confinement parameter is proportional to spectral radii of the flux Jacobian matrix. Therefore, the confinement parameter implicitly contains the local conditions of the flow field. This new method is named as lambda vorticity confinement method. In order to gain confidence in the applicability of vorticity confinement, it would be ideal to completely eliminate constant coefficients from confinement parameters. Because these constant coefficients should be determined for every problem by trial and error and it takes a long time. In the next part of this paper, a suitable relation is introduced for the vorticity confinement parameter that doesn't need any constant coefficient. This new method is named as adaptive vorticity confinement method. Then the capability of these new methods is compared with the other vorticity confinement methods for solving shock-vortex interaction and three dimensional moving vortex problems.
\end{abstract}

Keywords: Adaptive, Artificial dissipation, Numerical dissipation, Vorticity confinement.

\section{NOMENCLATURE}
A, B flux Jacobians $\partial \boldsymbol{f} / \partial \boldsymbol{Q}, \partial \boldsymbol{g} / \partial \boldsymbol{Q}$
D numerical viscosity
$e_{\text {。 }} \quad$ stagnation enthalpy per unit mass
$E_{c} \quad$ Scalar vorticity confinement parameter
$E_{c c} \quad$ Matrix vorticity confinement parameter
$E_{c L} \quad$ Lambda vorticity confinement parameter
$f \quad$ horizontal flux vector
$f_{b} \quad$ vector body force per unit mass
$g \quad$ vertical flux vector
$\hat{i}, \hat{j} \quad$ unit vectors in Cartesian coordinates
J Jacobian
$\mathrm{k}^{(2)}, \mathrm{k}^{(4)}$ small positive numbers
$\hat{\boldsymbol{n}}_{\boldsymbol{c}}$ unit vector in the direction opposite to the gradient of the vorticity magnitude
$p \quad$ pressure
$\boldsymbol{Q}$ vector of conservative variables
$R_{c} \quad$ vortex core radius
$R_{o} \quad$ vortex outer radius
$S \quad$ vorticity confinement vector

T matrix whose elements are composed of the eigenvectors of $\mathbf{A}$

$U_{c}$ vortex core velocity

$U_{\infty} \quad$ free stream velocity

$u, v$ Cartesian velocity components

$x, y \quad$ Cartesian coordinates

Greek Symbols

$\xi, \eta \quad$ Curvilinear coordinates

$\varepsilon_{x} \quad$ horizontal vorticity confinement parameter of adaptive method

$\varepsilon_{y} \quad$ vertical vorticity confinement parameter of adaptive method

$\lambda \quad$ spectral radii of $\mathbf{A}$

$\gamma \quad$ sensor

$\Delta \mathrm{V}$ volume of each cell

$\varepsilon^{(2)}, \varepsilon^{(4)}$ second and fourth order dissipation parameters for SCDS, MADS

$\Lambda$ diagonal matrix whose elements are the eigenvalues of $\mathbf{A}$

$\rho$ density

$\omega \quad$ vorticity vector 


\section{INTRODUCTION}

Many flows of interest are characterized by large regions of concentrated vortical structures that persist and can convect over long distances. Flows of this nature include those associated with aircrafts in particular rotorcrafts, ships, automobiles, bridges, and buildings. Conventional CFD methods tend to dissipate vortical structures, degrading the overall accuracy of the computed flows. This dissipation can be reduced through the use of fine grids, but at the expense of greatly increased computational demands.

Therefore, vorticity confinement method has been proposed to reduce the diffusive property of the incompressible and compressible vortical flow simulations by Steinhoff et al. (1992). In this method, the source term added to the Navier-Stokes equations works as it convects the discretization error back into the vortex center and thus confines the vortex. This has the effect of adding a velocity correction that convects vorticity in the opposite direction of the numerical diffusion (Malek Jafarian and Pasandideh Fard 2007).

However, in spite of the success of the method with regard to its goals, the confinement term is proportional to an empirical parameter $\left(E_{c}\right)$ that is constant over the entire flow field and has a dimension of velocity. Using a constant value over the entire flow field is questionable.

In order to make confinement parameter dependent on flow and computational parameters, several studies attempted different ideas: Fedkiw et al. (2001) introduced the mesh size $\mathrm{h}$ to guarantee the computational consistency with mesh refinement. Lohner and Yang (2002) devised a formulation where a proper length scale $h$ is computed by considering the gradient of vorticity magnitude and the confinement term is proportional to $h^{2}$. Robinson (2004) suggested a formulation where the confinement term is proportional to helicity. Malek Jafarian and Pasandideh Fard (2007) proposed new general formulations for the compressible confinement parameter which have the velocity dimension. Butsuntorn and Jameson (2008) devised a formulation where the confinement term is proportional to the logarithm of cell-volume ratio and helicity magnitude in their rotor-blade simulation using the time-spectral method.

In order to make confinement parameter dependent on flow and computational parameters, $\mathrm{Hu}$ et al. (2002) suggested a formulation that the confinement term was proportional to the flux Jacobian matrix. This method was called as matrix vorticity confinement method. But the calculation of flux Jacobian matrix is complicated and needs a long time in contrast to the other vorticity confinement methods. So a new confinement parameter is suggested in this paper which is proportional to spectral radii of the flux Jacobian matrix. This new confinement parameter has almost the accuracy of matrix vorticity confinement method and needs one half time of that. So this method is useful enough. This new confinement parameter is indicated with $E_{c L}$. The above mentioned studies introduce an improved dependency of the confinement terms on flow and mesh properties, but they don't completely eliminate the proportionality constant $\left(E_{c}\right)$.

Hahn and Iaccarino (2008) suggested a procedure for an improved prediction of vortical fields in the presence of numerical dissipation that was the adaptive vorticity confinement and the strength of the confinement term is computed without any need for an arbitrary constant. That formulation was suggested for incompressible flows. The Hahn and Iaccarino's formulation will be developed to the compressible vortical flow fields, in this paper.

\section{GOVERNING EQUATIONS FOR DIFFERENT VORTICITY CONFINEMENT METHODS}

Considering the Euler equations in Cartesian coordinates:

$$
\frac{\partial \boldsymbol{Q}}{\partial \mathrm{t}}+\frac{\partial \boldsymbol{f}}{\partial \mathrm{x}}+\frac{\partial \boldsymbol{g}}{\partial \mathrm{y}}=S
$$

where

$$
\begin{aligned}
& \boldsymbol{Q}=\left(\begin{array}{c}
\rho \\
\rho u \\
\rho v \\
\rho e_{\circ}
\end{array}\right), \quad \boldsymbol{f}=\left(\begin{array}{c}
\rho u \\
\rho u^{2}+P \\
\rho u v \\
\rho u h_{\circ}
\end{array}\right), \quad \boldsymbol{g}=\left(\begin{array}{c}
\rho v \\
\rho v u \\
\rho v^{2}+P \\
\rho v h_{\circ}
\end{array}\right) \\
& h_{\circ}=e_{\circ}+\frac{P}{\rho}, P=(\gamma-1) \times \rho \times\left\{e_{\circ}-\frac{u^{2}+v^{2}}{2}\right\} \\
& S=\left(\begin{array}{c}
0 \\
\rho f_{b} . \hat{\mathrm{i}} \\
\rho f_{b} \cdot \hat{\mathrm{j}} \\
\rho f_{b} . V
\end{array}\right)
\end{aligned}
$$

$\boldsymbol{Q}$ is the vector of conserved variables, $\boldsymbol{f}$ and $\boldsymbol{g}$ are the flux vectors and $\boldsymbol{S}$ is vorticity confinement vector. The independent variables are time $(t)$ and Cartesian coordinates $(x, y) . \quad \rho, u, v, e$ and $P$ denote nondimensional density, Cartesian velocity components, energy and pressure, respectively. $f_{b}$ is a body force per unit mass which tries to balance the numerical diffusion inherently related to numerical discretization and conserves momentum in vortical regions:

$$
f_{b}=-\mathrm{E}_{\mathrm{c}} \hat{n}_{c} \times \omega
$$

Where

$$
\begin{aligned}
& \hat{n}_{c}=-\frac{\nabla \varphi}{|\nabla \varphi|}, \varphi=|\omega|=\sqrt{\omega_{\mathrm{x}}{ }^{2}+\omega_{\mathrm{y}}{ }^{2}} \Rightarrow \hat{n}_{c}=\varphi_{\mathrm{xs}} \hat{\mathrm{i}}+\varphi_{\mathrm{ys}} \hat{\mathrm{j}} \\
& \varphi_{\mathrm{xs}}=\frac{\varphi_{\mathrm{x}}}{\sqrt{\varphi_{\mathrm{x}}{ }^{2}+\varphi_{\mathrm{y}}{ }^{2}}}, \varphi_{\mathrm{ys}}=\frac{\varphi_{\mathrm{y}}}{\sqrt{\varphi_{\mathrm{x}}{ }^{2}+\varphi_{\mathrm{y}}{ }^{2}}}
\end{aligned}
$$

Finally, the vorticity confinement term in a compressible flow can be expressed as follows: 


$$
\boldsymbol{S}=\left(\begin{array}{c}
0 \\
\rho f_{b x} \\
\rho f_{b y} \\
\rho\left(u f_{b x}+v f_{b y}\right)
\end{array}\right)=\left(\begin{array}{c}
0 \\
-\rho E_{c} \omega_{z} \varphi_{y s} \\
\rho E_{c} \omega_{z} \varphi_{x s} \\
-\rho E_{c} \omega_{z}\left(u \varphi_{y s}-v \varphi_{x s}\right)
\end{array}\right)
$$

Where $E_{c}$ is the scalar confinement parameter with the dimension of velocity. Also it is an empirical constant coefficient. $\omega_{z}$ is the z-component of the vorticity vector $(\omega)$. Assuming a constant coefficient for the scalar confinement parameter $\left(E_{c}\right)$ is questionable, because it has the dimension of velocity and should depend on the local conditions of flow field.

For solving this problem, the matrix confinement parameter was derived from the flux Jacobian matrix by Hu et al. (2001). The local conditions of flow field exist in the formulation of matrix vorticity confinement by flux Jacobian matrices. This is the preference of matrix vorticity confinement in contrast to the scalar vorticity confinement.

The matrix vorticity confinement term is defined as:

$$
\begin{aligned}
& \boldsymbol{S}=\boldsymbol{S}_{\mathrm{x}}+\boldsymbol{S}_{\mathrm{y}} \\
& S_{\mathrm{x}}=|\mathrm{A}|\left(\begin{array}{c}
0 \\
\mathrm{E}_{\mathrm{cc}} \rho\left(\hat{n}_{c} \times \omega\right) \cdot \hat{\mathrm{i}} \\
0 \\
\mathrm{E}_{\mathrm{cc}} \rho\left(\hat{n}_{c} \times \omega\right) \cdot \hat{\mathrm{i}}
\end{array}\right), S_{\mathrm{y}}=|\mathrm{B}|\left(\begin{array}{c}
0 \\
0 \\
\mathrm{E}_{\mathrm{cc}} \rho\left(\hat{n}_{c} \times \omega\right) \cdot \hat{\mathrm{j}} \\
\mathrm{E}_{\mathrm{cc}} \rho\left(\hat{n}_{c} \times \omega\right) \cdot \hat{\mathrm{j}}
\end{array}\right)
\end{aligned}
$$

Where $E_{c c}$ is the matrix confinement parameter with the dimension of velocity.

$|\mathbf{A}|$ and $|\mathbf{B}|$ are Jacobian matrices . $\mathbf{A}=\frac{\partial \boldsymbol{f}}{\partial \boldsymbol{Q}}$ and $|\mathbf{A}|$ is evaluated as $\mathbf{T} \boldsymbol{\Lambda} \mathbf{T}^{-1}$ with $\mathbf{T}$ a matrix whose columns are composed of the eigenvectors of $\mathbf{A}$ and $\boldsymbol{\Lambda}$ a diagonal matrix whose elements are eigenvalues of $\mathbf{A}$. The flux Jacobian matrix $\mathbf{B}$ is similarly defined as $\mathbf{B}=\frac{\partial \boldsymbol{g}}{\partial \boldsymbol{Q}}$ (Swanson and Turkel 1992). Althogh the local conditions of flow field exist in the formulation of matrix vorticity confinement by flux Jacobian matrices, but the calculation of flux Jacobian matrices needs a long time and great memory storage.

For solving this problem, we suggest a new formulation for vorticity confinement parameter. The flux Jacobian matrix is replaced with the spectral radii of flux Jacobian matrix in this new formulation. So the new confinement parameter has the following formulation:

$\mathrm{E}_{\mathrm{c}}=|\lambda| \times \mathrm{E}_{\mathrm{cL}}$

$|\lambda|$ is the spectral radii of flux Jacobian matrix and has the dimension of $\frac{m^{3}}{s}$. We describe about $|\lambda|$ at the next section. $E_{c}$ has the dimension of $\frac{m}{s}$. Thus according to Eq. (11), $E_{c L}$ has the dimension of $\frac{1}{\mathrm{~m}^{2}}$. If we use $E_{c}$ according to Eq. (11) in Eq. (8), the local conditions of flow field exist in the vorticity confinement term. This new vorticity confinement method is named as lambda method.

The accuracy of the lambda vorticity confinement method (Eq. 11) is almost equal to the matrix vorticity confinement method. But the required CPU time for the lambda method is almost one half of the matrix method. So the lambda formulation for vorticity confinement is an effective method.

Robinson (2004) carried out further dimensional analysis of confinement parameter for compressible flow and suggested the following formulation:

$S=\varepsilon \rho|u . \omega|\left\{\frac{\nabla|\omega|}{|\nabla| \omega \mid} \times \frac{\omega}{|\omega|}\right\}$

$\varepsilon$ is a dimensionless confinement parameter. According to Eq. (12), the vorticity confinement term $(\boldsymbol{S})$ is proportional to the magnitude of helicity ( $|\boldsymbol{u} . \boldsymbol{\omega}|)$.

The magnitude of helicity is equal to zero at two dimensional problems, thus the Robinson method is used at three dimensional problems.

Although the formulation provided by Robinson (2004) was an improvement over the previous works in some ways, it failed to take into account the length scale in the flow field. Thus Butsuntorn and Jameson (2008) tried to develop a new formulation for dynamic vorticity confinement method as an anti-diffusion term and proposed the following formulation:

$S=\varepsilon|\vec{u} \cdot \vec{\omega}|\left[\mathbf{1}+\log _{10}\left(1+\frac{\mathrm{V}}{\mathrm{V}_{\text {average }}}\right)^{\frac{1}{3}}\right]\left(\begin{array}{c}\mathbf{0} \\ \rho\left(\hat{n}_{c} \times \frac{\vec{\omega}}{|\vec{\omega}|}\right) \cdot \hat{\mathrm{i}} \\ \rho\left(\hat{n}_{c} \times \frac{\vec{\omega}}{|\vec{\omega}|}\right) \cdot \hat{\mathrm{j}} \\ \rho\left(\hat{n}_{c} \times \frac{\vec{\omega}}{|\vec{\omega}|}\right) \cdot \hat{\mathrm{k}} \\ \rho\left(\hat{n}_{c} \times \frac{\vec{\omega}}{|\vec{\omega}|}\right) \cdot \vec{u}\end{array}\right)$

Where $V$ is the volume of each cell in the grid and $V_{\text {average }}$ is the average volume of cells.

This formulation has the following properties:

1) The vorticity confinement term vanishes as the mesh size gets smaller $\left(\boldsymbol{S} \propto\left(\frac{V}{V_{\text {average }}}\right)^{\frac{1}{3}}\right)$. In other words the length scale of the grid exists in the vorticity confinement term.

2) Its strength is proportional to the gradient of the vorticity $(\boldsymbol{S} \propto \nabla|\omega|)$. 
The local conditions of flow field and the mesh size exist in Eq. (13). The vorticity confinement term is proportional to the magnitude of helicity, thus the Jameson method is used at three dimensional problems.

The conventional vorticity confinement methods have an empirical constant parameter (confinement parameter such as $\left.\left(E_{c}, E_{c c}, E_{c L}, \varepsilon\right)\right)$ that should be determined for every problem by trial and error. Different constant values should be examined for every problem and the best value should be selected for that problem. Selecting the best value for these constant coefficients needs a long time. In order to gain confidence in the applicability of vorticity confinement, it would be ideal to completely eliminate such constant parameters. Hahn and Iaccarino (2008) suggested a procedure for an improved prediction of incompressible vortical flow fields in the presence of numerical dissipation. That was the adaptive vorticity confinement, where the strength of the confinement term is computed without any need for an arbitrary constant. They proved this formulation by a balance between dissipation errors and vorticity confinement:

$$
\varepsilon\left(\mathrm{n}_{\mathrm{i}} \omega_{\mathrm{j}}-\mathrm{n}_{\mathrm{j}} \omega_{\mathrm{i}}\right)=\mathrm{e}_{\mathrm{ijk}} D_{\mathrm{k}}
$$

Where $D_{k}$ denotes the numerical viscosity.

As is used in the standard dynamic LES procedure (Lilly 1992) the least square error between both sides would lead to a good estimation for $\varepsilon$ and yields the following expression:

$$
\varepsilon=\frac{e_{i j k} D_{k} \times\left(n_{i} \omega_{j}-n_{j} \omega_{i}\right)}{\left(n_{i} \omega_{j}-n_{j} \omega_{i}\right) \times\left(n_{i} \omega_{j}-n_{j} \omega_{i}\right)}
$$

Hahn and Iaccarino (2008) estimated dissipation errors considering the difference between central and upwind discretizations of convection terms in the given flow field, as follows:

$\mathrm{D}_{\mathrm{i}} \approx \frac{\boldsymbol{\delta}^{\mathrm{CD}}}{\boldsymbol{\delta} \mathrm{x}_{\mathrm{j}}} \mathrm{u}_{\mathrm{i}} \mathrm{u}_{\mathrm{j}}-\frac{\boldsymbol{\delta}^{\mathrm{UD}}}{\boldsymbol{\delta} \mathrm{x}_{\mathrm{j}}} \mathrm{u}_{\mathrm{i}} \mathrm{u}_{\mathrm{j}}$

$\delta^{C D}, \delta^{U D}$ denote the central and upwind differences, respectively.

For 2- $D$ problems:

$$
\begin{gathered}
\varepsilon=\frac{\mathrm{D}_{1} \mathrm{n}_{2}-\mathrm{D}_{2} \mathrm{n}_{1}}{\omega_{3} \times\left(\mathrm{n}_{1}{ }^{2}+\mathrm{n}_{2}{ }^{2}\right)} \\
\mathrm{n}_{1}=\varphi_{\mathrm{xs}}, \mathrm{n}_{2}=\varphi_{\mathrm{ys}}
\end{gathered}
$$

$\varphi_{x s}$ and $\varphi_{y s}$ are calculated by using Eq. (7).

This formulation is developed for compressible flows in this part. The value of numerical viscosity at each point of the grid can be estimated by the value of artificial dissipation. So vorticity confinement parameters are introduced as follows:

$$
\begin{aligned}
& \varepsilon_{x}=\frac{D_{1} n_{2}-D_{2} n_{1}}{\omega_{3} \times\left(n_{1}{ }^{2}+n_{2}{ }^{2}\right)} \\
& \varepsilon_{y}=\frac{D_{3} n_{2}-D_{4} n_{1}}{\omega_{3} \times\left(n_{1}{ }^{2}+n_{2}{ }^{2}\right)}
\end{aligned}
$$

Where $D_{1}, D_{2}$ are the horizontal and vertical components of artificial dissipation term related to the x-momentum equation and $D_{3}, D_{4}$ are the horizontal and vertical components of artificial dissipation term related to the y-momentum equation. These variables are defined as follows:

$$
\begin{aligned}
& D_{1}=|\lambda|_{i+\frac{1}{2}, j}^{\left[\varepsilon^{(2)}\right.} \times\left(\boldsymbol{Q}_{i+1, j}{ }^{(2)}-\boldsymbol{Q}_{i, j}{ }^{(2)}\right)-\varepsilon^{(4)}{ }_{i+\frac{1}{2}, j}^{1} \times \\
& \left.\left(\boldsymbol{Q}_{i+2, j}{ }^{(2)}-3 \boldsymbol{Q}_{i+1, j}{ }^{(2)}+3 \boldsymbol{Q}_{i, j}{ }^{(2)}-\boldsymbol{Q}_{i-1, j}{ }^{(2)}\right)\right] \\
& D_{2}=|\lambda|_{i, j+\frac{1}{2}}\left[\varepsilon^{(2)} \times\left(\boldsymbol{Q}_{i, j+1}{ }^{(2)}-\boldsymbol{Q}_{i, j}{ }^{(2)}\right)-\varepsilon^{(4)} \times\right. \\
& \left.\left(\boldsymbol{Q}_{i, j+2}{ }^{(2)}-3 \boldsymbol{Q}_{i, j+1}{ }^{(2)}+3 \boldsymbol{Q}_{i, j}{ }^{(2)}-\boldsymbol{Q}_{i, j-1}{ }^{(2)}\right)\right] \\
& D_{3}=|\lambda|_{i+\frac{1}{2}, j}^{\left[\varepsilon^{(2)}\right.} \times\left(\boldsymbol{Q}_{i+1, j}{ }^{(3)}-\boldsymbol{Q}_{i, j}{ }^{(3)}\right)-\varepsilon^{(4)}{ }_{i+\frac{1}{2}, j} \times \\
& \left.\left(\boldsymbol{Q}_{i+2, j}{ }^{(3)}-3 \boldsymbol{Q}_{i+1, j}{ }^{(3)}+3 \boldsymbol{Q}_{i, j}{ }^{(3)}-\boldsymbol{Q}_{i-1, j}{ }^{(3)}\right)\right] \\
& D_{4}=|\lambda|_{i, j+\frac{1}{2}}\left[\varepsilon^{(2)} \times\left(\boldsymbol{Q}_{i, j+1}{ }^{(3)}-\boldsymbol{Q}_{i, j}{ }^{(3)}\right)-\varepsilon_{i, j+\frac{1}{2}}^{(4)} \times\right. \\
& \left.\left(\boldsymbol{Q}_{i, j+2}{ }^{(3)}-3 \boldsymbol{Q}_{i, j+1}{ }^{(3)}+3 \boldsymbol{Q}_{i, j}{ }^{(3)}-\boldsymbol{Q}_{i, j-1}{ }^{(3)}\right)\right]
\end{aligned}
$$

Bracketed superscripts for the vector $\boldsymbol{Q}$ in Eqs. (21) to (24) refer to the various components of this vector. So that $\boldsymbol{Q}_{i, j}{ }^{(2)}$ and $\boldsymbol{Q}_{i, j}{ }^{(3)}$ are the values of $\rho u$ and $\rho v$ at the point $(i, j)$, respectively.

These vorticity confinement parameters $\left(\varepsilon_{x}, \varepsilon_{y}\right)$ have the velocity dimension and they don't need any constant coefficient. They can be used at the following equation to determine the value of vorticity confinement at every point:

$$
\boldsymbol{S}=\left(\begin{array}{c}
0 \\
-\rho \varepsilon_{x} \omega_{z} \varphi_{y s} \\
\rho \varepsilon_{y} \omega_{z} \varphi_{x s} \\
-\rho \omega_{z}\left(u \varepsilon_{x} \varphi_{y s}-v \varepsilon_{y} \varphi_{x s}\right)
\end{array}\right)
$$

This new vorticity confinement method doesn't need any constant coefficient. Thus it is faster in contrast to the other vorticity confinement methods and it's an effective method. We call this new vorticity confinement method as adaptive method.

\section{NUMERICAL FORMULATION}

If Eq. (1) is transformed to arbitrary curvilinear coordinates, then we obtain:

$$
\hat{\boldsymbol{Q}}_{\mathrm{t}}+\hat{\boldsymbol{F}}_{\xi}+\hat{\boldsymbol{G}}_{\eta}=\boldsymbol{S}
$$

where 


$$
\begin{aligned}
& \hat{Q}=\mathbf{J}^{-1}\left[\begin{array}{c}
\rho \\
\rho \mathrm{u} \\
\rho \mathrm{v} \\
\mathrm{e}
\end{array}\right], \hat{F}=\mathbf{J}^{-1}\left[\begin{array}{c}
\rho \mathrm{U} \\
\rho \mathrm{uU}+\xi_{\mathrm{x}} \mathrm{P} \\
\rho \mathrm{vU}+\xi_{\mathrm{y}} \mathrm{P} \\
(\mathrm{e}+\mathrm{P}) \mathrm{U}
\end{array}\right] \\
& \hat{G}=\mathbf{J}^{-1}\left[\begin{array}{c}
\rho \mathrm{V} \\
\rho \mathrm{V}+\eta_{\mathrm{x}} \mathrm{P} \\
\rho \mathrm{V}+\eta_{\mathrm{y}} \mathrm{P} \\
(\mathrm{e}+\mathrm{P}) \mathrm{V}
\end{array}\right]
\end{aligned}
$$

and

$$
\mathrm{U}=\xi_{\mathrm{x}} \mathrm{u}+\xi_{\mathrm{y}} \mathrm{v}, \quad \mathrm{V}=\eta_{\mathrm{x}} \mathrm{u}+\eta_{\mathrm{y}} \mathrm{v}
$$

where $U$ and $V$ are contravariant velocities written without metric normalization and $\mathrm{J}^{-1}$ is the inverse transformation Jacobian. Then we obtain:

$$
\left(\mathbf{J}^{-1} \boldsymbol{Q}\right)_{t}+\boldsymbol{F}_{\xi}+\boldsymbol{G}_{\eta}=\boldsymbol{S}
$$

with

$$
\boldsymbol{F}=f \mathrm{y}_{\eta}-g \mathrm{x}_{\eta} \quad, \quad \boldsymbol{G}=\boldsymbol{g} \mathrm{x}_{\xi}-f \mathrm{y}_{\xi}
$$

In a cell-centered finite volume method, Eq. (29) is integrated over an elemental volume in the discretized computational domain, and $\mathrm{J}^{-1}$ is identified as the volume of the cell. Assuming $\mathrm{J}^{-1}$ to be independent of time, Eq. (29) can be written as follows:

$$
\mathbf{J}^{-1} \boldsymbol{Q}_{\mathrm{t}}+\mathbf{A} \boldsymbol{Q}_{\xi}+\mathbf{B} \boldsymbol{Q}_{\eta}=\boldsymbol{S}
$$

where $\mathbf{A}$ and $\mathbf{B}$ are the flux Jacobian matrices defined by:

$$
\mathbf{A}=\frac{\partial \boldsymbol{F}}{\partial \boldsymbol{Q}} \quad, \quad \mathbf{B}=\frac{\partial \boldsymbol{G}}{\partial \boldsymbol{Q}}
$$

To advance the solution in time the multi-stage scheme is applied. A typical step of a Runge-Kutta (5-stages) approximation to Eq. (31) is:

$$
\boldsymbol{Q}^{(k)}=\boldsymbol{Q}^{(0)}-\alpha_{k} \frac{\Delta t}{\Delta V}\left[\boldsymbol{D}_{\xi} \boldsymbol{F}^{(k-1)}+\boldsymbol{D}_{\eta} \boldsymbol{G}^{(k-1)}-\boldsymbol{S}-\boldsymbol{A D}\right]
$$

where

$\alpha_{k}=\left(\frac{1}{4}, \frac{1}{6}, \frac{3}{8}, \frac{1}{2}, 1\right)$

$\boldsymbol{D}_{\xi}$ and $\boldsymbol{D}_{\eta}$ are spatial differencing operators, and $\boldsymbol{A D}$ is the artificial dissipation term. $\Delta V$ is the volume of each cell and bracketed superscript in Eq. (33) refers to the stages of the Runge-Kutta scheme. In addition, local time stepping and implicit residual averaging are utilized to accelerate convergence.

The artificial dissipation schemes have been developed to remove the spurious oscillations for the robustness of stability and the fast convergence of the solutions in the steady-state aerodynamic problems. A combination of second and fourth differences of the flow variables is used to form the dissipation operator $\boldsymbol{A D}$. The second difference terms are used to prevent oscillations at shock waves, while the fourth difference terms are important in stability and convergence of the steady state solution.

Two common artificial dissipation schemes are as the following:

\section{SCalar Dissipation Scheme (SCDS)}

Where the dissipation model is divided into two terms in the $\xi$ and $\eta$ directions (Swanson and Turkel 1992). These two terms are written as:

$\boldsymbol{A D}=\hat{\boldsymbol{D}}_{\xi}+\hat{\boldsymbol{D}}_{\eta}$

where $\hat{\boldsymbol{D}}_{\xi}$ and $\hat{\boldsymbol{D}}_{\eta}$ are the dissipation terms in the $\boldsymbol{\xi}$ and $\eta$ directions, respectively. The dissipation term in the $\xi$ direction is defined as:

$\hat{\boldsymbol{D}}_{\xi}=\frac{\left(\hat{\boldsymbol{d}}_{\mathrm{i}+\frac{1}{2}, \mathrm{j}}-\hat{\boldsymbol{d}}_{\mathrm{i}-\frac{1}{2}, \mathrm{j}}\right)}{\Delta \xi}$

where the numerical dissipation flux at the horizontal direction is as follows:

$\hat{d}_{\mathrm{i}+\frac{1}{2}, \mathrm{j}}=|\lambda|_{\mathrm{i}+\frac{1}{2}, \mathrm{j}}\left[\varepsilon_{\mathrm{i}+\frac{1}{2}, \mathrm{j}}^{(2)} \times\left(Q_{\mathrm{i}+1, \mathrm{j}}-Q_{\mathrm{i}, \mathrm{j}}\right)-\varepsilon_{\mathrm{i}+\frac{1}{2}, \mathrm{j}}^{(4)} \times\right.$
$\left.\left(Q_{\mathrm{i}+2, \mathrm{j}}-3 Q_{\mathrm{i}+1, \mathrm{j}}+3 Q_{\mathrm{i}, \mathrm{j}}-Q_{\mathrm{i}-1, \mathrm{j}, \mathrm{j}}\right)\right]$

where $|\lambda|$ is proportional to the largest eigenvalue of the Jacobian matrix which is called the spectral radii of Jacobian matrix. The absolute eigenvalue is as the following:

$|\lambda|_{\mathrm{i}+\frac{1}{2}, \mathrm{j}}=\frac{1}{2}\left(|\lambda|_{\mathrm{i}+1, \mathrm{j}}+|\lambda|_{\mathrm{i}, \mathrm{j}}\right)$

where

$|\lambda|_{\mathrm{i}, \mathrm{j}}=\left|\boldsymbol{q}+\mathbf{c} \sqrt{\mathrm{a}_{1}^{2}+\mathrm{a}_{2}^{2}}\right|$

And

$\mathrm{a}_{1}=\mathbf{J}^{-1} \xi_{\mathrm{x}} \quad, \quad \mathrm{a}_{2}=\mathbf{J}^{-1} \xi_{\mathrm{y}} \quad, \quad q=\mathrm{a}_{1} \mathrm{u}+\mathrm{a}_{2} \mathrm{v}$

and $\mathrm{c}$ is the speed of sound. The nonlinear dissipation functions $\varepsilon^{(2)}$ and $\varepsilon^{(4)}$ in Eq. (37) determine $i+\frac{1}{2}, j \quad i+\frac{1}{2}, j$

the magnitude of the second and fourth order dissipation terms based on the density gradient.

$$
\begin{aligned}
& \varepsilon_{\mathrm{i}+\frac{1}{2} \mathrm{j}, \mathrm{k}}^{(2)}=\mathrm{k}^{(2)} \max \left(\gamma_{\mathrm{i}+1}, \gamma_{\mathrm{i}}\right) \\
& \varepsilon_{\mathrm{i}+\frac{1}{2} \mathrm{j}, \mathrm{k}}^{(4)}=\max \left(0, \mathrm{k}^{(4)}-\varepsilon_{\mathrm{i}+\frac{1}{2} \mathrm{j}, \mathrm{k}}^{(2)}\right)
\end{aligned}
$$

where $k^{(2)}$ and $k^{(4)}$ are small positive numbers and $\gamma_{i}$ is sensor. The role of the sensor is identification the regions near the shock waves, so that the high value of this variable indicates the regions near shock waves. In these regions the second order term is dominant. $\gamma_{i}$ is defined as follows: 


$$
\gamma_{i}=\frac{\left|\rho_{i+1, j, k}-\rho_{i, j, k}\right|-\left|\rho_{i, j, k}-\rho_{i-1, j, k}\right|}{\left|\rho_{i+1, j, k}-\rho_{i, j, k}\right|+\left|\rho_{i, j, k}-\rho_{i-1, j, k}\right|+\varepsilon}
$$

where $\varepsilon$ is a very small positive number to be used to avoid zero denominators in the smooth region.

We can write the numerical dissipation flux in the vertical direction as follows:

$$
\begin{aligned}
& \hat{\boldsymbol{d}}_{i, j+\frac{1}{2}}=|\lambda|_{i, j+\frac{1}{2}}\left[\varepsilon^{(2)} \times\left(\boldsymbol{Q}_{i, j+1}-\frac{1}{2}\right.\right. \\
& \left(\boldsymbol{Q}_{i, j}\right)-\varepsilon^{(4)} \\
& \left.\left(\boldsymbol{Q}_{i, j+2}-3 \boldsymbol{Q}_{i, j+1}+3 \boldsymbol{Q}_{i, j}-\boldsymbol{Q}_{i, j-1}\right)\right]
\end{aligned}
$$

The horizontal and vertical numerical dissipation fluxes $\left(\hat{\boldsymbol{d}}_{i+\frac{1}{2}, j}\right.$ and $\left.\hat{\boldsymbol{d}}_{i, j+\frac{1}{2}}\right)$ are $4 \times 1$ vectors for 2 -D problems. The first component of these vectors is related to the continuity equation. The second and third components are related to the $\mathrm{x}$ and $\mathrm{y}$ momentum equation, respectively and the fourth component of these vectors are related to the energy equation.

The SCalar Dissipation Scheme (SCDS) has an excellent shock capturing property and gives sufficient numerical stability to the central difference schemes.

\section{MAtrix Dissipation Scheme (MADS)}

The MADS scheme is very similar to the SCDS scheme, but the Jacobian matrix $\mathbf{A}$ is replaced instead of $|\lambda|_{i+\frac{1}{2}, j}$ in its formulation. (Swanson and Turkel 1992).

\section{Results AND Discussions}

In this part, different vorticity confinement methods are compared for two problems:

1) Shock- vortex interaction

2) Three dimensional moving vortex

As pointed earlier, the helicity term is equal to zero for two dimensional problems. Thus Robinson and Jameson methods are used for three dimensional moving vortex problem.

\subsection{Shock-Vortex Interaction}

We want to compute the problem of a moving shock over a circular vortex shown in Fig. 1. The Mach number of the shock wave is set to 1.5 .

An inviscid vortical velocity distribution between an outer radius $\left(r=R_{o}\right)$ and a core radius $\left(r=R_{c}\right)$ is prescribed for the vortex. Outside $R_{o}\left(r>R_{o}\right)$ the tangential velocity is set to zero. Inside the core ( $r<R_{c}$ ) the velocity goes linearly to zero at $r=0$. Thus the tangential velocity distribution is as follows:
$\mathrm{u}_{\theta}(\mathrm{r})=\left\{\begin{array}{lc}\frac{\mathrm{U}_{\mathrm{c}}}{\mathrm{R}_{\mathrm{c}}} & \mathrm{r} \leq \mathrm{R}_{\mathrm{c}} \\ \mathrm{Ar}+\frac{\mathrm{B}}{\mathrm{r}} & \mathrm{R}_{\mathrm{c}} \leq \mathrm{r} \leq \mathrm{R}_{\mathrm{o}}\end{array}\right.$

where

$A=-\frac{U_{c} R_{c}}{R_{o}{ }^{2}-R_{c}{ }^{2}}, B=\frac{U_{c} R_{o}{ }^{2} R_{c}}{R_{o}{ }^{2}-R_{c}{ }^{2}}$

The maximum tangential velocity of the circular vortex $\left(U_{c}\right)$ is 0.5 times the velocity behind the moving shock wave.

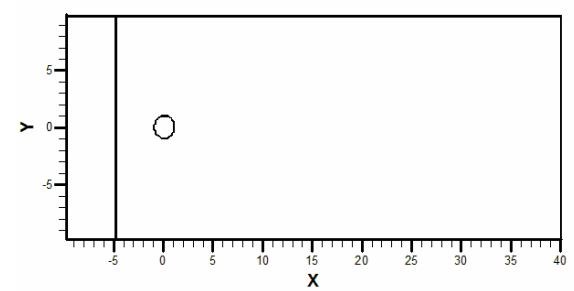

Fig. 1. A moving shock over a circular vortex

As we know, the inner region of the vortex has less density relative to the outer region. Thus the relative density is introduced as follows:

$\mathrm{D}=\frac{\rho_{\mathrm{L}}}{\rho_{\mathrm{h}}}<1$

Where $\rho_{L}$ is the density of region inside the vortex and $\rho_{h}$ is the density of region outside the vortex.

Some assumptions are considered to solve the problem as the following:

$R_{o}=2 R_{c}=1, D=0.2$

The computational grid is $50 \mathrm{R}_{\mathrm{o}} \times 20 \mathrm{R}_{\mathrm{o}}$. The grid is uniform along the $\mathrm{x}$-axis and is distributed according to an exponential function along the y-axis, so that the grid becomes finer as the y coordinate limits to zero.

Table 1 The time related to the different steps of shockvortex interaction

\begin{tabular}{|c|c|}
\hline Different steps & Time $(\mu \mathrm{s})$ \\
\hline $\mathrm{a}-1, \mathrm{~b}-1, \mathrm{c}-1$ & 50 \\
\hline $\mathrm{a}-2, \mathrm{~b}-2, \mathrm{c}-2$ & 70 \\
\hline $\mathrm{a}-3, \mathrm{~b}-3, \mathrm{c}-3$ & 90 \\
\hline $\mathrm{a}-4, \mathrm{~b}-4, \mathrm{c}-4$ & 110 \\
\hline $\mathrm{a}-5, \mathrm{~b}-5, \mathrm{c}-5$ & 157 \\
\hline $\mathrm{a}-6, \mathrm{~b}-6, \mathrm{c}-6$ & 203 \\
\hline
\end{tabular}

Figure 2 shows the density contours related to different steps of shock-vortex interaction. The density contours shown here are for two different grids $(1000 \times 200,300 \times 100)$ using scalar vorticity confinement $\left(E_{c}=0.02\right)$ and scalar artificial dissipation scheme. 
H. Bagheri Esfe et al. / JAFM, Vol. 5, No. 3, pp. 89-98, 2012.
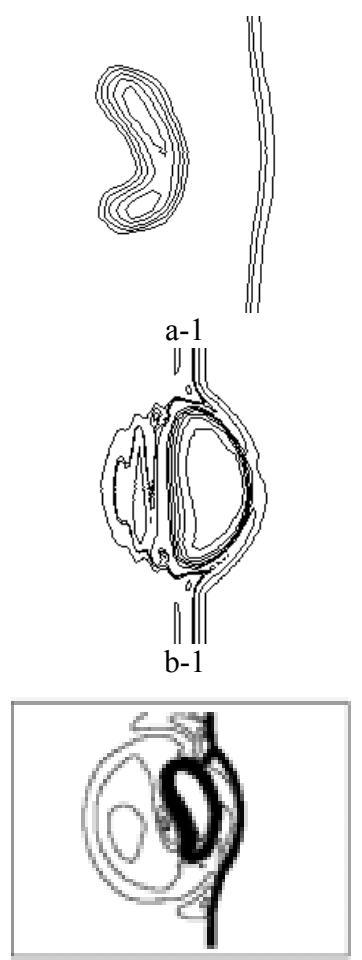

c-1

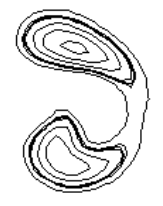

a-4

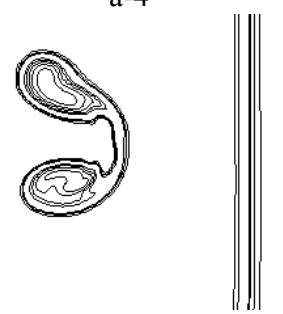

b-4

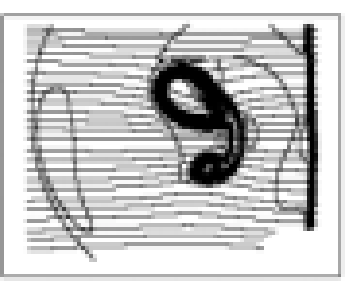

c-4

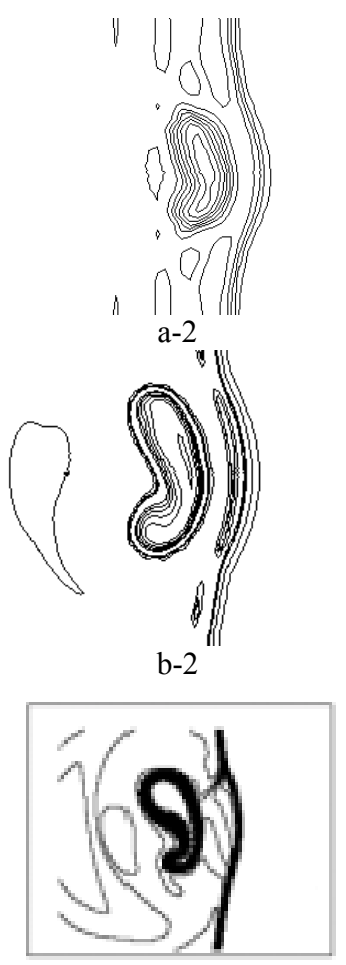

$\mathrm{c}-2$

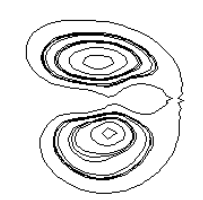

a-5

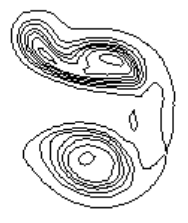

b-5

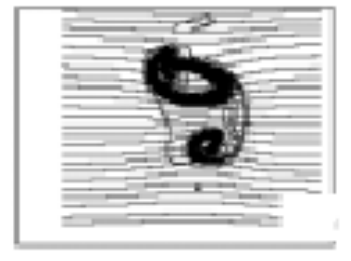

c-5

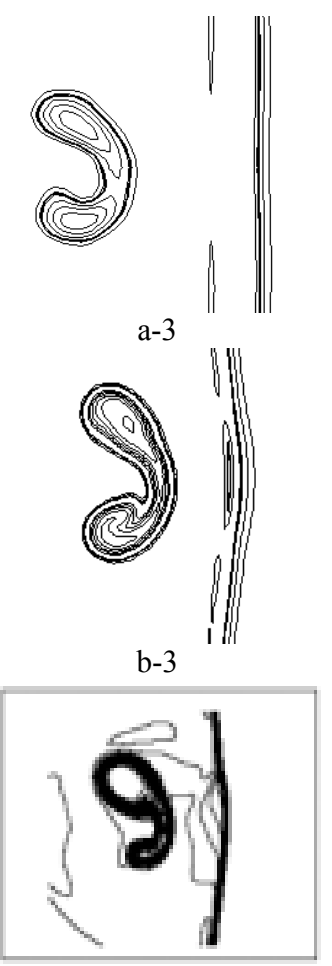

c-3
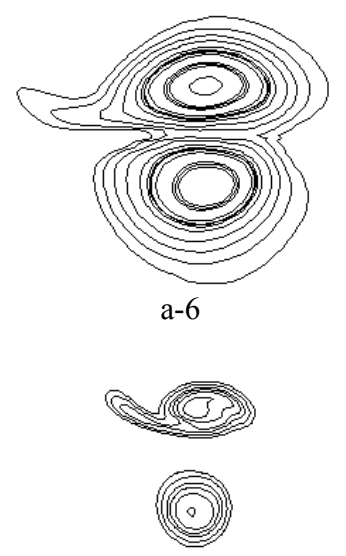

b-6

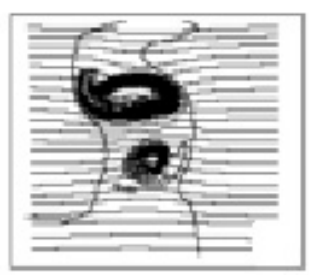

c-6

Fig. 2. Comparison the density contours related to $300 \times 1 \cdots$ grid: (a-1 to a-6) and $1000 \times$ r $\cdots$ grid: ( b-1 to b-6) with adaptive unstructured algorithm by Povitsky et al. (1998): (c-1 to c-6)

They are compared to the adaptive unstructured algorithm results of Povitsky et al. (1998), in Fig. 2. This figure shows the results of different time steps. These time steps are shown in Table 1. The results are similar and they get closer to the adaptive unstructured algorithm results as the grid becomes finer
$(1000 \times 200)$. If we simulate the shock-vortex interaction using different vorticity confinement methods, the overall shapes for various steps of interaction are almost similar but they haven't shown here to prevent repetition. 
To compare the different vorticity confinement methods, it would be better to find the values of a variable (such as pressure) at the core of main and secondary vortices. Figure 3 shows the vorticity contours at time $\mathrm{t}=421 \mu \mathrm{s}$.

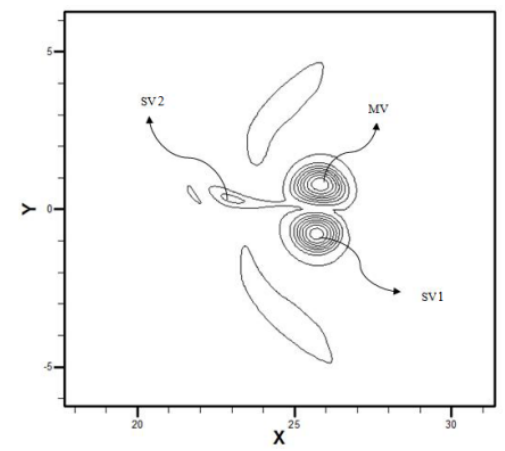

Fig. 3. Vorticity contours related to shock-vortex interaction at time $\mathrm{t}=421 \mu \mathrm{s}$

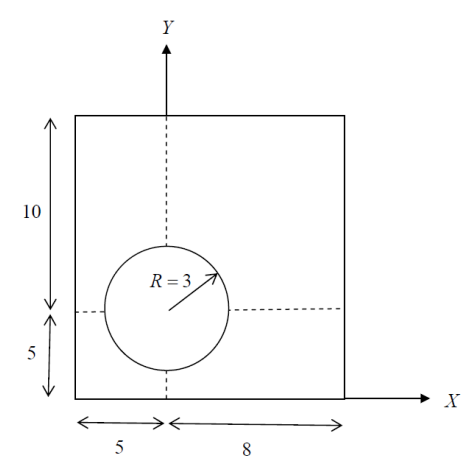

Fig. 4. Two dimensional section for the vortex and its domain

Three different regions are specified in this figure: MV (Main vortex), SV1 (Secondary vortex 1) and SV2 (Secondary vortex 2).

Table 2 compares the pressure in the center of three vortices of the present study with different vorticity confinement methods and a fine grid $(1000 \times 200)$. The results related to the coarser grid $(300 \times 100)$ are shown in Table 3. Also $P_{a m b}$ denotes the ambient pressure in these tables. The scalar vorticity confinement method with confinement parameter $E_{c}$, the new lambda confinement method with confinement parameter $E_{c L}$ and the new adaptive method are compared in these tables. As we know, whatever the vorticity in the center of the vortex becomes larger, the pressure will be smaller, if the ambient conditions are fixed.

Thus Tables 2 and 3 show that the pressure in the center of three regions reduces with vorticity confinement (W/ CVC), relative to the without vorticity confinement (W/O CVC) state. The most accurate method among three different vorticity confinement methods studied here, is the lambda method with $E_{c L}=0.2$ in Tables 2 and 3.
Because the results of lambda method are closer to the results of Povitsky et al. (1998) in contrast to the other vorticity confinement methods. As pointed earlier, the local conditions of flow field exist in the lambda formulation (according to Eq. (11)), thus this method has the best answer in contrast to the others. The results get better as the grid becomes finer $(1000 \times 200)$. Also the adaptive method is less accurate in contrast to the other vorticity confinement methods, but as pointed earlier its real worth is eliminating empirical constant coefficient ( such as $E_{c}, E_{c c}, E_{c L}$ ). Thus this method is faster in contrast to the other vorticity confinement methods.

\subsection{Three Dimensional Moving Vortex}

As pointed earlier, Robinson and Jameson vorticity confinement methods have a term (helicity term) that is equal to zero at two dimensional problems. Thus we compare the capability of these methods with the other vorticity confinement methods for three dimensional moving vortex problem. For making the three dimensional vortex and its domain, we consider the two dimensional section in Fig. 4. If this section rotates along the $\mathrm{x}$-axis, the vortex and its cylindrical domain are made. A $100 \times 100 \times 21$ grid is considered for the domain. The Mach number of the moving vortex is set to 0.8 . Thus we consider a specific moving vortex at the position $\mathrm{x}=0$. After 15000 time steps, the vortex reaches the position $x=2.73$. The vorticity contours related to these positions are presented in Fig. 5.

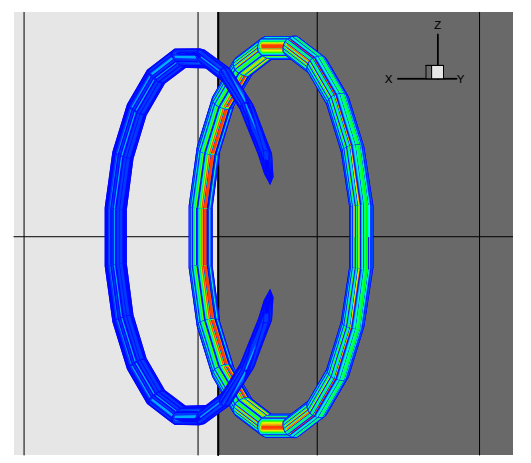

Fig. 5. The initial situation of vortex (the right contours)and the situation of the vortexafter 15000 time steps ( the left contours), without vorticity confinement

Dissipation of the vortex after moving to the left side is obvious in this figure. We can use the vorticity confinement methods to reduce these numerical dissipations. To compare the capability of different vorticity confinement methods, it would be better to compare the vorticity contours at a cross section of the vortex. The results are shown in Fig. 6.

Degradation of vorticity contours after 15000 time steps, is obvious in this figure. The numerical dissipations decrease significantly if we use the Jameson vorticity confinement method with $\varepsilon=0.03$ (Fig. 7). 
Table 2 The pressure comparison $\left(\frac{P}{P_{a m b}}\right)$ for different vorticity confinement methods and the grid $(1000 \times 200)$

\begin{tabular}{|c|c|c|c|c|c|}
\hline $\begin{array}{c}\text { Metho } \\
\mathrm{d}\end{array}$ & $\begin{array}{c}\text { W/O } \\
\text { CVC }\end{array}$ & $\begin{array}{c}\text { New adaptive } \\
\text { method }\end{array}$ & $\begin{array}{c}\text { W/ CVC } \\
\left(E_{c}=0.02\right)\end{array}$ & $\begin{array}{c}\text { W/ CVC } \\
\left(E_{c L}=0.2\right)\end{array}$ & $\begin{array}{c}\text { Adaptive algorithm by } \\
\text { Povitsky et al. (1998) }\end{array}$ \\
\hline Grid & $1 \cdots-r \cdots$ & $1 \cdots-r \cdots$ & $1 \cdots-r \cdots$ & $1 \cdots-r \cdots$ & Unstructured grid \\
\hline MV & 0.935 & 0.929 & 0.913 & 0.893 & 0.884 \\
\hline SV1 & 0.895 & 0.894 & 0.892 & 0.889 & 0.89 \\
\hline SV2 & 1.007 & 1.006 & 1.003 & 1.001 & 0.993 \\
\hline
\end{tabular}

Table 3 The pressure comparison $\left(\frac{P}{P_{a m b}}\right)$ for different vorticity confinement methods and the grid $(300 \times 100)$

\begin{tabular}{|c|c|c|c|c|c|}
\hline $\begin{array}{c}\text { Metho } \\
\mathrm{d}\end{array}$ & $\begin{array}{c}\text { W/O } \\
\text { CVC }\end{array}$ & $\begin{array}{c}\text { New adaptive } \\
\text { method }\end{array}$ & $\begin{array}{c}\text { W/ CVC } \\
\left(E_{c}=0.02\right)\end{array}$ & $\begin{array}{c}\text { W/ CVC } \\
\left(E_{c L}=0.2\right)\end{array}$ & $\begin{array}{c}\text { Adaptive algorithm by } \\
\text { Povitsky et al. (1998) }\end{array}$ \\
\hline Grid & $\mu \cdots-1 \cdots$ & $r \cdots-1 \cdots$ & $r \cdots-1 \cdots$ & $r \cdots-1 \cdots$ & Unstructured grid \\
\hline MV & 0.969 & 0.958 & 0.946 & 0.936 & 0.884 \\
\hline SV1 & 0.954 & 0.931 & 0.902 & 0.895 & 0.89 \\
\hline SV2 & 1.008 & 1.007 & 1.005 & 1.003 & 0.993 \\
\hline
\end{tabular}

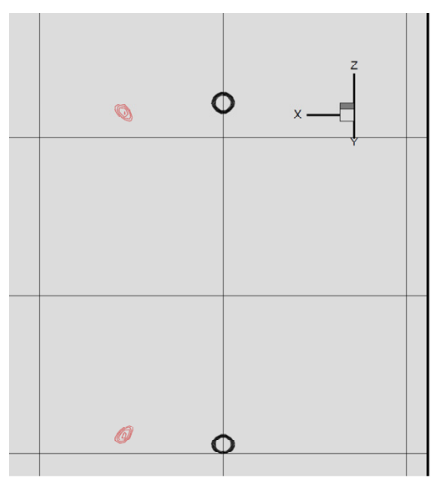

Fig. 6. Two dimensional vorticity contours related to the initial vortex (the right contours)and the vortex after 15000 time steps ( the left contours), without vorticity confinement

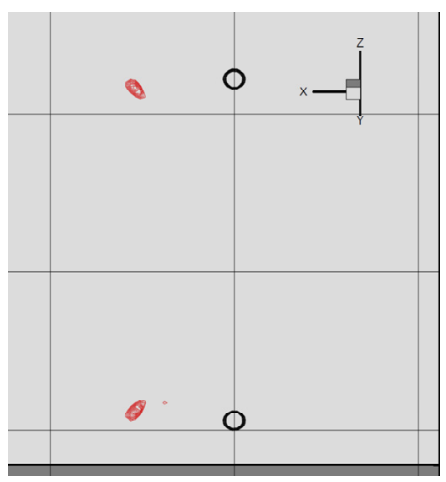

Fig. 7. Two dimensional vorticity contours related to the initial vortex (the right contours)and the vortex after 15000 time steps ( the left contours), Jameson vorticity confinement method with $\varepsilon=0.03$
The two dimensional vorticity contours related to other vorticity confinement methods are almost similar to Fig. 7, but the magnitude of vorticity in the center of two dimensional vortices is different for every method. The magnitude of vorticity in the core region of two dimensional vortices for different vorticity confinement methods are shown in Table 4 . The results are related to the initial vortex $(\mathrm{nct}=1)$ and the vortex after 15000 time steps (nct=15000).

Table 4 The magnitude of vorticity in the center of 2D vortices for two states of vortex,

Comparison between different vorticity confinement methods for the $100 \times 100 \times 21$ grid

\begin{tabular}{|c|c|c|c|}
\hline \multirow{2}{*}{ Method } & \multirow{2}{*}{$E_{c}$} & \multicolumn{2}{|c|}{ Vorticity } \\
\cline { 4 - 5 } & & nct=1 & nct=15000 \\
\cline { 1 - 1 } Without confinement & $\cdot$ & 6.54 & 3.52 \\
\cline { 1 - 1 } Scalar & 0.03 & 6.54 & 4.46 \\
\cline { 1 - 1 } Jameson & 0.03 & 6.54 & 5.05 \\
\cline { 1 - 1 } Robinson & 0.03 & 6.54 & 4.80 \\
\cline { 1 - 1 } New adaptive method & - & 6.54 & 3.76 \\
\cline { 1 - 1 } Lambda & 0.05 & 6.54 & 4.91 \\
\hline
\end{tabular}

This table shows that the Jameson (with $\varepsilon=0.03$ ) and the lambda vorticity confinement methods (with $E_{c L}=0.05$ ) are more accurate in contrast to the other methods. Because these methods conserve the initial vorticity more than the other methods. As pointed earlier the local conditions of flow field exist in these two formulations. Thus these methods (Jameson and 
lambda) are more affective in contrast to the other methods. Also Table 4 shows that the Robinson method is more accurate in contrast to the scalar method for conserving the initial vorticity. Also the adaptive method is less accurate than the other vorticity confinement methods. But this method doesn't need any constant coefficient for the confinement parameter.

Table 5 The magnitude of vorticity in the center of 2D vortices for two states of vortex,

Comparison between different vorticity confinement methods for the $200 \times 200 \times 21$ grid

\begin{tabular}{|c|c|cc|}
\hline \multirow{2}{*}{ Method } & \multirow{2}{*}{$E_{c}$} & \multicolumn{2}{|c|}{ Vorticity } \\
\cline { 4 - 4 } & & nct=1 & nct=15000 \\
\hline Without confinement & $\cdot$ & 6.54 & 4.45 \\
\cline { 1 - 1 } Scalar & 0.03 & 6.54 & 5.37 \\
\cline { 1 - 1 } Jameson & 0.03 & 6.54 & 6.01 \\
\cline { 1 - 1 } Robinson & 0.03 & 6.54 & 5.83 \\
\cline { 1 - 1 } New adaptive method & - & 6.54 & 4.85 \\
\cline { 1 - 1 } Lambda & 0.05 & 6.54 & 5.89 \\
\hline
\end{tabular}

The results related to another grid $(200 \times 200 \times 21)$ have been shown in Table 5. Comparing the Tables 4 and 5 shows that the results will be more accurate for a finer grid.

\section{Conclusion}

In this paper, a new vorticity confinement method (lambda method) was introduced using spectral radii of the flux Jacobian matrix. The results related to two different problems showed that this new vorticity confinement method is effective enough. Also this formulation (lambda) is faster than the matrix vorticity confinement method.

Also the Hahn and Iaccarino's (2008) method was extended to compressible flows (adaptive method). This method doesn't need any empirical constant coefficient. The results showed that this method has less accuracy in contrast to the other vorticity confinement methods, but its real worth is eliminating empirical constant coefficients. So this method (adaptive method) is faster than the other vorticity confinement methods and thus it is an effective method.

In the next part of this paper, we compare the capability of Jameson and Robinson methods with the other vorticity confinement methods using the three dimensional moving vortex problem. The results showed that the Jameson and lambda methods are more accurate in contrast to the other vorticity confinement methods. Because the local conditions of flow field exist in the formulation of these methods. The results showed that the Robinson method has more accuracy relative to the scalar method and has less accuracy in contrast to the Jameson and lambda methods.

\section{REFERENCES}

Butsuntorn, N. and A. Jameson (2008). Time spectral method for rotorcraft flow with vorticity confinement. AIAA Paper-0403.

Fedkiw, R., J. Stam and W. Jensen (2001). Visual simulation of smoke. International Conference on Computer Graphics and Interactive Techniques, $15-22$.

Hahn, S. and G. Iaccarino,(2008). Simulations of vortex-dominated flows: adaptive vorticity confinement vs. rotational-form upwinding. Center for Turbulence Research, Annual Research Briefs.

Hu, G., B. Grossman and J. Steinhoff (2002). A numerical method for vortex confinement in compressible flows. AIAA paper- 0714, 40, 19451953.

Hu, G (2001). The Development and Applications of a Numerical Method for Compressible Vorticity Confinement in Vortex-Dominant Flows. $\mathrm{PhD}$ Thesis, Dep Of Aerospace and Ocean Engineering, Virginia Polytechnic Inst. And State Univ, Blacksburg, VA.

Lilly, D.K. (1992). A proposed modification of the Germano sub grid-scale closure method. Phys. Fluids A 4, 633-635.

Lohner, R. and C. Yang (2002). Tracking vortices over large distances using vorticity confinement. 24th Symposium on Naval Aerodynamics, Fukuoka, Japan.

Malek Jafarian, M. and M. Pasandideh Fard (2007). Development and application of compressible vorticity confinement. Scientia Iranica Journal 14, 251-262.

Povitsky, A. and D. Ofengeim (1998). Numerical Study of Interaction of a Vortical Density Inhomogeneity With Shock and Expansion Waves. NASA/CR-206918, ICASE Report, No. 98-10.

Robinson, A (2004). Application of vorticity confinement to inviscid missile force and moment prediction. AIAA Paper-0717.

Steinhoff, J., C. Wang, D. Underhill, T. Mersch and Y. Wenren (1992). Computational vorticity confinement: A non-diffusive Eulerian method for vortex dominated flows. Univ. of Tennessee Space Inst., Preprint, Tullahoma, TN.

Swanson, R. and E. Turkel (1992). On central difference and upwind schemes. Journal of computational physics 101, 292-306. 